\author{
Disponible on-line en: \\ http://revistalogopedia.uclm.es \\ Revista de Investigación en Logopedia 1 (2015) 1-17. \\ ISSN-2174-5218
}

UNIVERSIDAD DE

\title{
Comprensión lectora, habilidades lingüísticas y decodificación en escolares con TEL
}

\author{
Carmen Julia Coloma, Carmen Sotomayor, Zulema De Barbieri y \\ Macarena Silva
}

1 Universidad de Chile, Chile

\begin{abstract}
Resumen
El propósito del presente trabajo es indagar sobre la comprensión lectora y su relación con la decodificación y con algunas habilidades lingüísticas (léxico, conciencia fonológica y discurso narrativo) en escolares con TEL. Para ello, se trabajó con 104 niños de $1^{\circ}$ básico con un promedio de edad de 6 años 8 meses, que se distribuyeron en dos grupos: uno conformado por 51 escolares con TEL y el otro constituido por 53 niños con desarrollo típico de lenguaje (DT). Las habilidades que se evaluaron fueron léxico, conciencia fonológica, narración (comprensión y producción), decodificación y comprensión lectora. Los resultados mostraron que en los niños con TEL la comprensión lectora se correlacionó significativamente con el léxico y la decodificación. En el grupo con DT, además de advertir las mismas asociaciones, también se observó una relación entre conciencia fonológica y comprensión lectora. Los resultados confirman que la decodificación es una habilidad central para la comprensión lectora. Además, el nivel léxico aparece como la única habilidad lingüística que se asocia a la lectura comprensiva en el aprendizaje lector inicial de los niños con TEL.

Palabras clave: Lectura, decodificación, trastorno específico del lenguaje, comprensión lectora, habilidades lingüísticas
\end{abstract}

Reading comprehension, linguistics abilities and decodification in young students with SLI

\begin{abstract}
The purpose of this study is to investigate the relationship between decoding and some language skills (vocabulary, phonological awareness and narrative discourse) with reading comprehension in students with specific language impairment (SLI). To do this, we worked with 104 first grade students with an average of 6 years 8 months, which were distributed into two groups: one was made up of 51 school children with SLI and the other consisted of 53 children with typical language development (TLD). The skills assessed were vocabulary, phonological awareness, narrative (comprehension and production), decoding and reading comprehension. The results showed that the reading comprehension of children with SLI was significantly correlated with vocabulary and decoding. In the group with TLD the same associations were noted, although a relationship between phonological awareness and reading comprehension was also observed. The results confirm that decoding is a core skill for reading comprehension. In addition, the vocabulary appears as the only linguistic skill associated with comprehensive reading in the initial reading learning of children with SLI.
\end{abstract}

Keywords: Reading, decoding, specific language impairment, reading comprehension, linguistics skills

Correspondencia con los autores: ccoloma@ med.uchile.cl. Recibido: 20 octubre 2014. Primera revisión 2 diciembre 2014. Aceptado 23 Diciembre 2104 Agradecimientos: Se agradece el financiamiento otorgado por el FONDECYT Regular 2013 nº1130201 de la Comisión Nacional de Investigación y Tecnología (CONICYT) y por el Proyecto Basal FB0003 del Programa de Investigación Asociativa de CONICYT, Ministerio de Educación, Chile. 


\section{Introducción}

El Trastorno Específico del Lenguaje (TEL) se define como un déficit en el desarrollo lingüístico que se produce en ausencia de factores que puedan explicarlo (Leonard, 1998). Por lo anterior, se efectúa un diagnóstico por exclusión para asegurar que no existan dificultades auditivas, neurológicas, cognitivas ni ambientales que causen el problema (Fresneda \& Mendoza, 2005).

Además de sus problemas lingüísticos, muchos de los niños con TEL exhiben dificultades lectoras que pueden estar influidas por un menor rendimiento del nivel léxico, de la conciencia fonológica y del discurso, consideradas habilidades relevantes para el desempeño lector (Ricketts, 2011, Nation \& Snowling, 2004; Coloma \& De Barbieri, 2007; Dickinson, McCabe, Anastasopoulos, Peisner-Feinberg \& Poe, 2003).

En relación al léxico, los niños con TEL presentan dificultades para aprender nuevos términos. Por esta razón, adquieren las primeras palabras más tardíamente que los menores con desarrollo típico (Gray, 2003); su léxico es general y poco diverso (Leonard \& Deevy, 2004). Además, manifiestan problemas en la denominación y en el uso de palabras funcionales (Buiza, Adrián, González \& Rodríguez-Parra, 2004).

Los déficits en conciencia fonológica en los menores con TEL se producen tanto en la etapa pre-escolar como en la escolar, lo que se advierte en que les es difícil analizar y sintetizar la palabra oral a nivel de la sílaba y del fonema (De Barbieri \& Coloma, 2004; Webster \& Plante, 1992). Es destacable que la emergencia de la conciencia fonológica requiere de la adquisición de una masa crítica de vocabulario (Goswami, 2001), por lo tanto, estas habilidades presentan grados de dependencia.

También los menores con TEL manifiestan problemas en diferentes ámbitos del discurso narrativo, tales como la estructura textual, la coherencia y los mecanismos de cohesión (Pearce, James \& McCormack, 2010).

Por otra parte, la perspectiva del modelo de la Visión Simple de Lectura es un marco de referencia útil para entender las dificultades lectoras de los niños con TEL. Este modelo da cuenta de las diferencias individuales en comprensión lectora y plantea que ésta es producto de la decodificación y de la comprensión oral. La decodificación es entendida como el eficiente reconocimiento de la palabra escrita, lo que implica la adecuada asociación entre la representación fonológica y el input de las palabras impresas. La comprensión oral es similar a la comprensión lectora, sin embargo difieren en la presentación del material, que en el primer caso es impreso y en el segundo, oral. Dentro de los componentes de comprensión oral destaca el vocabulario, ya que aporta 
una varianza adicional en la comprensión lectora, una vez controladas otras medidas de comprensión oral (Protopapas, Simos, Sideridis \& Mouzaki, 2012).

El hecho de que la comprensión lectora sea producto de la decodificación y la comprensión oral implica que ambas habilidades son necesarias para lograr una lectura comprensiva (Hoover \& Gough, 1990). Es decir, la comprensión lectora es imposible si no existen grados mínimos tanto de decodificación como de comprensión oral (Protopapas et al, 2012). Además, este modelo propone que el impacto de estas habilidades varía durante el proceso de aprendizaje de la lectura. Así, mientras la decodificación se está adquiriendo, ésta es fundamental para la comprensión lectora. En cambio, cuando la decodificación se automatiza, la comprensión oral se vuelve esencial para que se comprenda lo que se lee.

Por otra parte, la decodificación y la comprensión lectora están basadas en habilidades diferentes. Así, la conciencia fonológica constituye la base de la decodificación mientras que el vocabulario, la gramática y el discurso narrativo sustentan la comprensión lectora (Oakhill \& Cain, 2012). Desde esta perspectiva, las dificultades lectoras de los niños con TEL podrían estar determinadas por sus déficits fonológico, léxico y discursivo. De este modo, los problemas fonológicos afectarían a la decodificación y los déficits, tanto léxicos como discursivos, impactarían en la comprensión lectora (Bishop \& Snowling, 2004).

El planteamiento previo implica que los menores con TEL deberían presentar desempeños disminuidos en decodificación y en comprensión lectora; sin embargo, este fenómeno no siempre ocurre. De hecho, se ha observado que algunos escolares con TEL no tienen dificultades lectoras.

Un estudio longitudinal reportó que en segundo básico el 67\% de escolares con TEL evidenció dificultades lectoras y en cuarto básico dichas dificultades disminuyeron al 63.7\% (Catts, Fey, Tomblin \& Zhang, 2002). Resultados similares se encontraron en otro estudio donde se observó que el 67\% de escolares con TEL de primero básico manifestaron dificultades en comprensión lectora (Coloma, Pavez, Peñaloza, Araya, Maggiolo \& Palma, 2012). Por otra parte, se ha advertido que algunos alumnos con TEL presentan exclusivamente dificultades en comprensión lectora y otros, solo manifiestan compromiso en decodificación (Kelso, Fletcher \& Lee, 2007; Bishop McDonald, Bird \& Hayiou-Thomas, 2009). Las investigaciones previas muestran la heterogeneidad del desempeño lector en estos niños. Lo anterior indica que la lectura y su relación con los déficits lingüísticos en los niños con TEL aún requieren de mayor estudio. 
Un ámbito relevante es la importancia de la decodificación y de las habilidades lingüísticas en la comprensión lectora de los escolares que inician su aprendizaje lector. Ello debido a que en las etapas iniciales, la decodificación debería estar fuertemente asociada a la comprensión lectora. No obstante, en los niños con TEL este fenómeno podría presentarse de manera diferente.

En una investigación se encontró que ni la decodificación ni la narración se relacionaron con la comprensión lectora en niños de primero básico con TEL (Coloma et al., 2012). En cambio, en otro estudio se observó que los escolares con TEL que no estructuraban sus narraciones orales presentaban dificultades lectoras, lo que sugiere que la producción narrativa tiene algún nivel de impacto en el desempeño lector de estos alumnos (Coloma \& Alarcón, 2009).

Los estudios mencionados se han centrado solo en el discurso narrativo y su relación con la comprensión lectora. Sin embargo, existen otras habilidades lingüísticas, como el léxico y la conciencia fonológica, que también podrían asociarse a la comprensión lectora y que no han sido suficientemente investigados en niños con TEL hablantes del español.

Por esto, los objetivos del presente trabajo son: a) determinar la relación entre habilidades lingüísticas (léxico, conciencia fonológica y discurso narrativo) y comprensión lectora en escolares de primero básico con TEL y b) determinar la relación entre decodificación y comprensión lectora en estos mismos escolares.

\section{Método}

La investigación que se presenta es un estudio correlacional porque indaga sobre las posibles relaciones que se pueden encontrar entre comprensión lectora, habilidades lingüísticas y decodificación.

\section{Participantes}

En esta investigación participaron 104 escolares de primero básico con un promedio de edad de seis años, ocho meses. Los niños se distribuyeron en dos grupos: uno conformado por 51 niños con TEL y otro por 53 escolares con desarrollo típico de lenguaje (DT). Es importante destacar que los padres de los participantes habían aceptado que sus hijos fueran parte del estudio mediante la firma de un consentimiento informado.

Asistían a escuelas municipales o particulares subvencionadas con proyectos de integración de la Región Metropolitana de Santiago de Chile. Los colegios pertenecen a 
los grupos socioeconómicos $\mathrm{A}$ y $\mathrm{B}^{1}$ (promedio de escolaridad de los padres entre 8 y 10 años, media de ingreso familiar mensual entre US\$285 y US\$420).

Por su parte, los proyectos de integración son programas que ofrecen los establecimientos educacionales para apoyar a los niños con necesidades educativas especiales.

Todos debían presentar un desempeño en habilidades cognitivas no verbales y en audición dentro de rangos típicos. Con este fin se aplicó el test de Matrices Progresivas, Escala Coloreada para evaluar las habilidades cognitivas (Raven, 2005) y una audiometría de barrido.

El test de Matrices Progresivas es una prueba que emplea la modalidad no verbal y, por ello, es aplicable a cualquier persona, independiente de su idioma, educación y capacidad lingüística. Según el test, las puntuaciones ubicadas en el percentil 25 o sobre éste se interpretan como normales. Por lo tanto, se incluyó en el estudio a aquellos niños que puntuaron dentro del rango normal definido por esta prueba. Se realizó una comparación de medias con una t de Student que indicó que no hubo diferencias significativas en las habilidades cognitivas no verbales entre ambos grupos (Media TEL: 19.68 y Media DT: 20.84 (t 1.96, p=0.052)).

Para la audiometría de barrido se usó un audiómetro clínico marca Interacoustic modelo AD629 en las frecuencias 500, 1000, 2000 y 4000 Hz. Se utilizaron los criterios internacionales propuestos por la ASHA que determinan la normalidad auditiva a intensidades iguales o inferiores a $20 \mathrm{~dB}$ (American Speech-Language-Hearing Association, 2005).

El grupo con TEL se seleccionó a partir del diagnóstico que realizó el profesional fonoaudiólogo del centro educativo. Dicho diagnóstico se basa en criterios estándares propuestos por el Ministerio de Educación de Chile que indican que para establecer el diagnóstico de TEL, el niño debe presentar rendimientos deficitarios en comprensión y/o en expresión del lenguaje, según pruebas normalizadas en Chile (Decreto Supremo, 170/2010). Los escolares con TEL que participaron en este estudio presentaban dificultades tanto a nivel expresivo como comprensivo del lenguaje.

En cuanto al grupo control, los 53 menores con DT fueron seleccionados entre los compañeros de curso de los escolares con TEL. Para corroborar su desarrollo típico, las

\footnotetext{
${ }^{1}$ Clasificación socioeconómica de los estudiantes, definida por el Sistema de Medición de la Calidad de la Educación (SIMCE) de Chile, donde A representa el nivel socioeconómico bajo y B el nivel medio bajo.
} 
examinadoras revisaron la ficha escolar, confirmando que este grupo no presentara antecedentes de problemas de lenguaje o de aprendizaje. Además, solicitaron a la profesora seleccionar a niños con un rendimiento escolar de nivel medio, de modo de evitar incluir estudiantes con desempeños muy destacados o muy descendidos.

\section{Instrumentos}

A continuación se presentan los instrumentos para evaluar tanto las habilidades lingüísticas como las lectoras.

\section{Habilidades Lingüísticas}

\section{Conciencia Fonológica}

Esta habilidad metalingüística se estudió mediante la "Prueba de Conciencia Fonológica para $1^{\circ}$ básico" (Himmel \& Infante, 2007). Consta de 8 subpruebas que contienen 6 ítems cada una. Las dos primeras son actividades que requieren comparar fonemas, tanto iniciales como finales; las subpruebas tercera y cuarta implican aislar fonemas iniciales y finales; la quinta supone realizar síntesis fonémica; la sexta y la séptima exigen omitir el fonema inicial o final. Por último, la octava subprueba demanda segmentar fonemas de la palabra escuchada. A cada respuesta correcta se le asigna 1 punto, por lo tanto, el máximo puntaje en esta prueba es 48. Su confiabilidad es .96 y se obtuvo mediante el coeficiente $\alpha$ Cronbach.

\section{Narración}

Se estudió la comprensión y producción narrativa con el instrumento "Evaluación del Discurso Narrativo (EDNA)" (Pavez et al., 2008) que contiene dos subpruebas para cada modalidad. A continuación, se exponen las características por separado de ambas subpruebas.

\section{Producción narrativa}

El método EDNA implica el recontado de tres cuentos de distinta complejidad estructural. Los relatos son: La ardillita glotona, El sapito saltarín y El lobo friolento. Los cuentos infantiles se analizan identificando tres categorías estructurales básicas (presentación, episodio y final) a las que se les asigna puntaje. Se establece si las categorías están completas o incompletas según los elementos que las conforman: presentación completa $($ personaje + problema $=1$ punto $) ;$ presentación incompleta $($ personaje o problema $=0.5)$; 
episodio completo (acción + obstáculo + resultado $=2$ puntos); episodio incompleto (acción + obstáculo u obstáculo + resultado $=1$ punto $)$ y final $=1$ punto.

Cuando no es posible identificar las categorías en los relatos, el método propone una sistematización para analizar las narraciones a partir de los siguientes desempeños: no dice

nada ( 0 punto), dice una o dos oraciones ( 0.25 puntos), aglutina tres o más oraciones sin relación ( 0.5 puntos), agrupa tres o más oraciones en torno a un personaje ( 0.75 puntos) o produce secuencia acción + obstáculo u obstáculo + resultado (0.75 puntos).

Es necesario destacar que esta prueba no tiene un puntaje máximo, ya que la puntuación asignada depende del desempeño narrativo logrado por el niño. La prueba de producción presenta una confiabilidad de .77

\section{Comprensión narrativa}

La prueba de comprensión de EDNA incluye tres cuestionarios, uno para cada cuento, con preguntas inferenciales y literales (La ardillita glotona: 6 preguntas literales y 3 inferenciales, El sapito saltarín y El lobo friolento: 7 preguntas literales y 3 inferenciales). Tanto las interrogantes literales como las inferenciales se relacionan con las categorías estructurales. Se asigna 1 punto a las respuestas literales, 2 puntos a las que requieren inferencias, en consecuencia, el puntaje máximo es de 38 puntos y la de comprensión de .84 según el coeficiente $\alpha$ de Cronbach.

Las pruebas mencionadas anteriormente no utilizan criterio de corte.

\section{Léxico}

Se utilizó el instrumento "Vocabulario sobre dibujos", que corresponde a la batería "Language Survey -Revised" (versión en español) de Woodcock, Muñoz, Ruef y Alvarado (2005). Está compuesto por 58 ítems que aumentan progresivamente en dificultad en la medida en que las palabras evaluadas son menos familiares para los niños. Cada ítem está ilustrado por una imagen que representa el término evaluado. Pese a que la prueba mide vocabulario en las modalidades de comprensión y producción, cabe destacar que 52 ítems corresponden al léxico expresivo. En el caso del léxico comprensivo, se identifica la imagen de la palabra ilustrada en la lámina mientras que en el léxico expresivo se nomina el término representado en la imagen.

Las respuestas correctas se valoran en 1 punto, en consecuencia, la máxima puntuación es 58 puntos. La prueba para evaluar léxico presenta una confiabilidad de .89, de acuerdo a la prueba de Kuder y Richardson. 


\section{Habilidades lectoras}

Las habilidades lectoras consideradas fueron decodificación y comprensión lectora. La primera se midió mediante la prueba "Identificación de letras y palabras" y la segunda, a través de la prueba "Comprensión de textos", ambos de la batería "Language Survey Revised" (versión en español) de Woodcock, Muñoz, Ruef y Alvarado (2005). Los aspectos generales de cada prueba se describen continuación.

\section{Identificación de letras y palabras}

Está constituida por 75 ítems, de los cuales los primeros corresponden a la identificación de letras, mientras que los siguientes refieren a palabras que se deben leer fluidamente. La complejidad de los ítems está determinada por su grado de familiaridad. Es decir, las palabras son progresivamente menos frecuentes en la medida en que el test avanza.

Las respuestas correctas son valoradas con 1 punto y la puntuación máxima es de 75 puntos. La prueba de Identificación de letras y palabras presenta una confiabilidad de .96 , de acuerdo a la prueba de Kuder y Richardson.

\section{Comprensión lectora}

Esta prueba mide compresión de palabras, oraciones y textos. Comienza con ítems que evalúan la habilidad de relacionar la palabra con un dibujo del objeto que ella representa. En los siguientes ítems se asocia un dibujo a una frase. Posteriormente, se presentan oraciones con distinto grado de complejidad sintáctica que requieren ser completadas con una palabra. Luego, aparecen textos progresivamente más difíciles por su sintaxis y léxico, los cuales también deben ser completados con una palabra que les otorgue sentido. Es importante destacar que para realizar las tareas de esta prueba se requiere de un buen manejo de la decodificación.

A las respuestas correctas se les asigna 1 punto y la máxima puntuación que se puede alcanzar es 32 puntos. La prueba de Comprensión de textos presenta una confiabilidad de .95 , según la prueba de Kuder y Richardson.

Las pruebas de vocabulario, decodificación y comprensión lectora son parte de una misma batería, por lo que tienen criterios similares para su utilización. Se aplican por páginas completas y se suspenden cuando el menor presenta un número de errores consecutivos según las indicaciones de cada prueba. 


\section{Procedimiento de evaluación}

La evaluación se realizó individualmente en una sala tranquila y silenciosa en el establecimiento educacional del menor. La aplicación de los instrumentos se organizó en dos momentos del año. En el primer semestre, se evaluaron las habilidades lingüísticas y en el segundo, se midieron las habilidades lectoras.

Las habilidades lingüísticas se evaluaron en dos sesiones. La primera destinada al léxico y al discurso narrativo. En la segunda sesión se aplicó la prueba para evaluar conciencia fonológica. Las habilidades lectoras también se evaluaron en dos momentos. El primero se orientó a la evaluación la decodificación y el segundo estuvo dirigido a la medición de la comprensión lectora. Es importante señalar que las sesiones no sobrepasaron los 15 minutos con el fin de que el menor mantuviera un nivel de atención adecuado.

En las pruebas de discurso narrativo EDNA, se le pidió al niño que escuchara los cuentos y que después los narrara. Se leyó cada narración con naturalidad y sin apoyo visual en el siguiente orden: primero La ardillita glotona, luego El sapito saltarín y, finalmente, El lobo friolento. Después de escuchar cada cuento, el escolar los relató y su narración fue grabada. Posteriormente, se le formularon las preguntas y se registraron sus respuestas.

La aplicación de la prueba de "Vocabulario sobre dibujos", implicó que el niño mostrara (comprensión léxica) o nominara (expresión léxica) la palabra correspondiente a una imagen que fue señalada por el examinador.

La evaluación de conciencia fonológica requirió que el examinador produjera la palabra estímulo correspondiente a cada subprueba y luego el niño debía realizar la tarea específica solicitada.

En la prueba de "Identificación de letras y palabras" el menor tenía que identificar letras en distintos contextos: entre dibujos, entre otras letras o entre números. Luego, debía reconocer palabras, leer términos progresivamente más complejos en su fonología, en su ortografía y en su significado.

Finalmente, para evaluar la comprensión lectora se pidió al niño que relacionara un pictograma con una ilustración. Luego, se le solicitó que mostrara la ilustración correspondiente a una frase leída por él. Finalmente, el menor debía leer y completar oraciones y textos breves donde faltaba una palabra. 


\section{Resultados}

El objetivo de este estudio busca correlacionar la comprensión lectora con habilidades lingüísticas y de decodificación, para esto se utilizó el coeficiente de correlación $r$ de Pearson. Se exponen primero los resultados descriptivos y comparativos con el fin de ilustrar las características de ambos grupos. Para efectuar la comparación, se aplicó la prueba t de Student.

Los estadísticos descriptivos y la comparación entre niños TEL y DT se presentan en la Tabla 1. Todas las variables se comportaron de forma adecuada, con excepción de la conciencia fonológica que presentó una curtosis elevada. Se realizó, por lo tanto, una transformación logarítmica para normalizar dicho puntaje.

Tabla 1. Comparación de los desempeños en habilidades lingüísticas y lectoras entre niños TEL y niños DT

\begin{tabular}{cccccccc}
\hline \multicolumn{1}{c}{ Variables } & \multicolumn{2}{c}{ TEL } & \multicolumn{2}{c}{ DT } & & \\
\hline & Media & DS & Media & DS & $t$ & $p^{a}$ \\
\hline Habilidades lingüísticas & & & & & & \\
Conciencia fonológica & 8.14 & 5.30 & 17.92 & 9.76 & 6.39 & .00 \\
Comprensión narrativa & 23.0 & 6.5 & 27.1 & 5.0 & 3.59 & .00 \\
Producción narrativa & 7.6 & 4.9 & 9.2 & 4.6 & 1.74 & .08 \\
& & & & & & \\
$\quad$ Léxico & 28.9 & 5.0 & 32.3 & 4.0 & 3.88 & .00 \\
Habilidades lectoras & & & & & & \\
$\quad$ Decodificación & 32.3 & 18.8 & 52.2 & 16.3 & 5.79 & .00 \\
Comprensión lectora & 7.1 & 4.3 & 13.2 & 4.7 & 6.79 & .00 \\
\hline Diferencia es significativa si p $<0.05$ & & & & & &
\end{tabular}

La Tabla 1 muestra que el grupo con TEL presenta un rendimiento significativamente inferior al grupo control en la mayoría de las habilidades evaluadas. Esto es en decodificación, comprensión lectora, conciencia fonológica, comprensión narrativa y léxico. La habilidad lingüística en que no hubo diferencias significativas fue la producción narrativa. Sin embargo, el grupo con TEL tuvo un promedio inferior al grupo de niños sin problemas lingüísticos. Debido a que no se encontraron diferencias significativas, se determinó qué porcentaje de niños con TEL y con DT evidenciaban problemas en la producción de narraciones. Para la identificación de los niños con problemas narrativos, se utilizó el criterio propuesto en EDNA (Pavez et al., 2008). Este consiste en que los menores con puntajes que se ubican en el percentil 10 o inferior evidencian dificultades narrativas. Así, se determinó que el 57\% de los participantes con 
TEL manifestaba dificultades narrativas, en cambio, el $49 \%$ del grupo con DT evidenciaba déficit en este aspecto.

En la Tabla 2 se presentan las correlaciones de la comprensión lectora con las habilidades lingüísticas y con la decodificación en los niños con TEL y en los menores con DT.

Tabla 2. Correlaciones entre comprensión lectora y conciencia fonológica, léxico, comprensión narrativa, producción narrativa y decodificación en niños con TEL y niños DT

\begin{tabular}{|c|c|c|}
\hline & & \\
\hline & TEL & DT \\
\hline $\begin{array}{l}\text { Conciencia } \\
\text { Fonológica }\end{array}$ & .266 & $.384^{* * *}$ \\
\hline Léxico & $.296^{*}$ & $.460^{* *}$ \\
\hline $\begin{array}{l}\text { Comprensión } \\
\text { narrativa }\end{array}$ & .043 & .384 \\
\hline Producción narrativa & .157 & . -113 \\
\hline Decodificación & $.875 * *$ & $.795 * *$ \\
\hline
\end{tabular}

En la Tabla 2 se observa que, en los niños con TEL, la comprensión lectora se correlaciona con el léxico y con la decodificación. Es necesario destacar que la asociación con la decodificación es la más alta. Por su parte en el grupo con DT, el léxico y la conciencia fonológica se asociaron a la comprensión lectora. Al igual que el grupo con TEL, se encontró relación significativa con la decodificación.

\section{Discusión}

Los análisis de correlación mostraron un comportamiento similar entre los niños con TEL y los menores con DT, lo que se advirtió en varios de los resultados obtenidos. Así, en ambos grupos, la relación entre decodificación y comprensión lectora fue la más alta. Además, tanto en los niños con TEL como en los menores con DT se observó asociación entre el léxico y la comprensión lectora. También hubo coincidencia en la ausencia de correlación del discurso narrativo (comprensión y producción) con la lectura comprensiva. 
La única diferencia fue que en los niños con TEL no se relacionó la comprensión lectora con la conciencia fonológica, fenómeno que sí se produjo en el grupo con DT.

La relación encontrada entre decodificación y comprensión lectora es consistente con la propuesta del modelo de la Visión Simple de la Lectura, ya que plantea que, en las etapas iniciales del aprendizaje lector, la decodificación juega un rol central en la comprensión de textos (Catts \& Kamhi, 2005). Por lo tanto, es factible esperar una importante asociación entre decodificación y comprensión lectora en niños que inician su aprendizaje lector.

También la relación entre léxico y comprensión lectora es esperable, porque un amplio vocabulario es requisito fundamental para lograr entender lo leído. De hecho, se ha establecido que el vocabulario aporta una varianza adicional en la comprensión lectora, una vez controladas otras medidas de comprensión oral (Protopapas, Simos, Sideridis \& Mouzaki, 2012).

Además, la decodificación y el léxico son habilidades que se requieren entre sí. Esto, debido a dos razones: una se basa en que la decodificación contribuye al aumento y afianzamiento del léxico, ya que el ejercicio de la lectura incrementa las entradas en el léxico mental a través de la ruta fonológica y, posteriormente, mediante la ruta léxica o directa. Esta última estrategia, utilizada por los lectores competentes, posibilita que una palabra sea reconocida, porque concuerda con su representación almacenada en la memoria léxica (Reynoso-Alcantara et al., 2010). La otra razón, refiere al hecho de que decodificar implica la habilidad para derivar rápidamente una representación desde la palabra escrita que posibilite acceder a una apropiada entrada al léxico mental y así recuperar la información semántica (Hoover \& Gough, 1990). En consecuencia, los sujetos deben disponer de un léxico mental variado para lograr reconocer diversas palabras escritas. Como lo indican varios autores, cuantas más palabras familiares posea el lector, más directamente reconocerá el término impreso (Alegría, 1989; Stanovich, 1989; Morais et al., 1991).

Desde esta perspectiva, se puede proponer la hipótesis de que, en las etapas iniciales del aprendizaje lector, el vocabulario se asocia a la comprensión lectora, porque contribuye al proceso de identificación de la palabra escrita.

Por otra parte, no hubo relación entre desempeño narrativo (comprensión y producción) y comprensión lectora, lo que sugiere que cuando la tarea de lectura requiere necesariamente de la decodificación. Habilidades lingüísticas como la comprensión y la producción narrativa tienen una menor relevancia, lo que significa que a los niños con un 
bajo nivel de decodificación se les dificulta enormemente realizar la tarea de comprender un texto escrito.

Lo anterior se sustenta en que la lectura comprensiva es un constructo que abarca una serie de procesos cognitivos que no son directamente observables. Por ello, los resultados difieren dependiendo de la modalidad utilizada en la evaluación de la comprensión. Así, la comprensión lectora está altamente relacionada con la decodificación cuando la tarea de comprensión solo se logra si se decodifica. En cambio, cuando la actividad de comprensión lectora se puede realizar sin requerir tanto de la decodificación, las asociaciones entre lectura comprensiva y habilidades linguiísticas, como la narración, son más altas (Ricketts, 2011).

La ausencia de relación entre comprensión lectora y conciencia fonológica en los niños con TEL podría deberse a que ellos obtuvieron disminuidos puntajes en la prueba que evaluó esta habilidad metalingüística. Además, la conciencia fonológica es un factor relevante en la decodificación, por lo que sería más esperable que su relación con la comprensión lectora se diera vía la decodificación.

Las comparaciones, realizadas permitieron constatar que los niños con TEL presentan un desempeño significativamente inferior al del grupo con DT en la mayoría de las habilidades lingüísticas estudiadas (comprensión narrativa, léxico y conciencia fonológica). Estos resultados son consistentes con estudios previos donde se ha establecido que los niños con TEL presentan dificultades en los distintos componentes del lenguaje (Leonard \& Deevy, 2004; Nation \& Snowling, 2004; Pearce, James \& McCormack, 2010).

La única habilidad en que no se encontraron diferencias significativas fue la producción de narraciones. Una posible explicación a este resultado puede ser el hecho de que muchos niños con DT evidenciaron un desempeño descendido en este tipo de discurso. En otro trabajo tampoco se encontraron diferencias significativas en narraciones entre niños con antecedentes de problemas de lenguaje y menores con desarrollo típico, pareados por edad cronológica. Al igual que en la presente investigación, el grupo control evidenció bajos rendimientos en este tipo de discurso (Domsch, Richels, Saldana, Coleman, Wimberly \& Maxwel, 2012).

En consecuencia, la ausencia de diferencias encontradas en ambas investigaciones sugiere que los problemas en el discurso narrativo no son exclusivos de los niños con dificultades lingüísticas. Por lo tanto, es posible que existan menores con desarrollo típico que manifiesten deficiencias en la producción de sus relatos, en especial, menores 
pertenecientes al nivel socioeconómico bajo. Al respecto, existe evidencia que muestra que algunos niños con desarrollo típico y deprivación sociocultural presentan desempeños narrativos descendidos (Contreras \& Soriano, 2004).

Finalmente, los resultados referidos a las comparaciones de las habilidades lectoras muestran que el grupo con TEL evidencia un rendimiento significativamente inferior al del grupo con DT. Lo anterior corrobora la idea de que los niños con este trastorno lingüístico son un grupo de riesgo para el aprendizaje lector.

\section{Conclusiones}

Los hallazgos encontrados señalan que en las primeras etapas del aprendizaje lector de los niños, la decodificación y el vocabulario se relacionan con la comprensión lectora. Esto sugiere que ambas habilidades serían centrales para lograr una adecuada comprensión de los textos escritos.

Los datos indicaron que una alta asociación entre decodificación y comprensión lectora se produce cuando la tarea de lectura comprensiva exige manejar eficientemente la decodificación. Además, los resultados señalaron que el grupo con TEL presentaba un disminuido desempeño en decodificación. En consecuencia, en especial en estos niños, se debe realizar un trabajo sistemático para favorecer la decodificación en las primeras etapas de su aprendizaje lector.

También es necesario abordar el vocabulario ya que, al comparar ambos grupos, se advirtió que los menores con TEL presentaban un rendimiento inferior al de sus pares con DT. Además, fue la única medida lingüística que se asoció a la comprensión lectora. Esto sugiere que, junto a la decodificación se debería apoyar el vocabulario no solo para incrementar el lenguaje oral, sino también para aportar a la comprensión lectora de los menores que exhiben este trastorno lingüístico.

Por último, los resultados están limitados por el instrumento para evaluar comprensión lectora porque, como ya se mencionó, la prueba utilizada exige la automatización de la decodificación. Por lo tanto, dificulta la posibilidad de observar el comportamiento de otras variables lingüísticas en la lectura comprensiva. Por ello, sería muy interesante estudiar las relaciones entre las habilidades lingüísticas y la comprensión lectora con un instrumento de lectura comprensiva que no dependa exclusivamente del dominio de la decodificación. Esto permitiría evaluar con mayor precisión el impacto del lenguaje oral en la comprensión lectora de los escolares con TEL. 


\section{Referencias}

Alegría, J. (1989). Mécanismes d'acquisition de la lecture: perspective cognitive. Séminaire du « recyclage inter-reseaux P.M.S ». Ministère de l'Éducation Nationale. Bruxelles : ULB.

American Speech-Language-Hearing Association (2005). Audiology Information Series [en línea]. Disponible en: [http://www.asha.org/uploadedFiles/aud/InfoSeriesHearingLossTypes.pdf\#searc

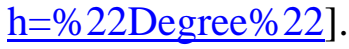

Bishop, D., y Snowling, M. (2004). Developmental Dyslexia and Specific Language Impairment: Same or Different? Psychological Bulletin, 130, 858-886.

Bishop, D., McDonald, D., Bird, S. y Hayiou-Thomas, M. (2009). Children who read words accurately despite language impairment: Who are they and how do they do it? Child Development, 80, 593-605.

Buiza, J. Adrián, J. González, M., y Rodríguez-Parra, M. (2004). Evaluación de marcadores psicolingüísticos en el diagnóstico de niños con Trastorno Específico del Lenguaje. Revista de Logopedia, Foniatría y Audiología, 24, 142-155.

Catts, H., Fey, M.,Tomblin ,J.B., y Zhang, X. (2002). A longitudinal Investigation of Reading Outcomes in Children with Language Impairments. Journal of Speech, Language, and Hearing Research, 45, 1142-1157.

Catts, H., y Kamhi, A. (2005). Language and Reading Disabilities. Boston: Pearson

Coloma, C.J. y De Barbieri, Z. (2007) Trastorno Fonológico y conciencia fonológica en preescolares con Trastorno Específico del Lenguaje. Revista de Logopedia, Foniatría y Audiología, 27, 67-73.

Coloma, C.J., y Alarcón, P. (2009). El discurso narrativo y la comprensión lectora en escolares con Trastorno Específico del Lenguaje. Revista de Psicología General y Aplicada, 62,147-158.

Coloma, C.J., Pavez, M.M., Peñaloza, C., Araya, C., Maggiolo, M. y Palma, S. (2012). Desempeño lector y narrativo en escolares con trastorno específico del lenguaje. Revista Onomazein, 26, 351-375.

Contreras, M.C., y Soriano, M. (2004). El valor de la narrativa en la caracterización de los alumnos con dificultades de lenguaje. Revista de Logopedia, Foniatría y Audiología, 24, 119-125.

De Barbieri, Z., y Coloma, C.J. (2004). La conciencia fonológica en niños con TEL. Revista de Logopedia, Foniatría y Audiología, 24, 156 -163. 
Dickinson, D., McCabe, A., Anastasopoulos, L., Peisner-Feinberg, E., y Poe, D. (2003). The Comprehensive Language Approach to Early Literacy: The Interrelationships among Vocabulary, Phonological Sensitivity, and Print Knowledge Among Preschool-Aged Children. Journal of Educational Psychology, 95 (3), 465-481.

Domsch, C., Richels, C., Saldana, M., Coleman, C., Wimberly, C., y Maxwel, L. (2012). Narrative skill and syntactic complexity in school-age children with and without late language emergence. International Journal of Language \& Communication Disorders, 47, 197-207.

Fresneda, D., y Mendoza, E. (2005).Trastorno Específico del lenguaje: concepto, calcificaciones y criterios de identificación. Revista de Neurología, 41, 51-56.

Goswami, U. (2001). Early phonological development and the acquisition of literacy. En S. B. Neuman y D. K. Dickinson (Eds.), Handbook of early literacy research. (pp.111-125). New York: Guilford Press.

Gray, S. (2003). Word-Learning by preschoolers with Specific Language Impairment: What predicts Success? Journal of Speech, Language, and Hearing Research, 46, 56-67.

Himmel, E., e Infante, M. (2007). Construcción y validación del perfil de aprendizajes logrados en los sectores de lenguaje y matemáticas en los niveles NB1 y NB2. Proyecto FONDECYT No 1040985. Facultad de Educación de la Pontificia Universidad Católica de Chile.

Hoover, W., y Gough, Ph. (1990). The Simple View of Reading. Reading and Writing: An interdisciplinary Journal, 2, 127-160.

Kelso, K., Fletcher, J., y Lee, P. (2007). Reading comprehension in children with specific language impairment: an examination of two subgroups. International Journal of Language \& Communication Disorders, 42, 39-57.

Leonard, L. (1998). Children with Specific Language Impairment. Cambridge: MIT Press.

Leonard, L., y Deevy, P. (2004). Lexical deficits in specific language impairment. En L. Verhoeven y H. van Balkom (Eds.), Classification of developmental language disorders. Theoretical issues and clinical implications. (pp.209-233). New Jersey London: Lawrence Erlbaum Associates, publishers.

Ministerio de Educación (2010). Decreto Supremo, 170/2010, Ley 20201, Unidad de Educación Especial. 
Morais, J., Castro, S.L., y Kolinsky, R. (1991). La reconnaissance des mots chez les adultes illettrés. En R. Kolinsky, J. Morais y J. Segui (Eds.), La reconnaissance des mots dans les différentes modalités sensorielles: études de psycholinguistique cognitive. (pp. 59-80). Paris: Presse Universitaires de France.

Nation, K. y Snowling, M. (2004). Beyond phonological skills: broader language skills contribute to the development of reading. Journal of Research in Reading, 27, $342-356$.

Oakhill, J. y Cain, K. (2012). The precursors of reading ability in young readers: Evidence from a four-year longitudinal study. Scientific Studies of Reading, 16, 91-12.

Pávez, M.M., Coloma, C.J.. y Maggiolo, M. (2008). El desarrollo narrativo en niños. Barcelona: Ars Médica.

Pearce, W., James, D., y Mc Cormack, P. (2010). A comparison of oral narratives children with specific language and non-Specific language impairment. Clinical Linguistics \& Phonetics, 24, 622-646.

Protopapas, A., Simos, P. Sideridis, G., y Mouzaki, A. (2012). The components of the simple view of reading: a confirmatory factor analysis. Reading Psychology, 33, $217-240$.

Reynoso-Alcántara, V., Bernal, J., Silva-Pereyra, J.S., Rodríguez, M., Yáñez, G., Fernández, T. y Del Río, Y. (2010). Procesamiento fonológico y léxico en niños lectores de educación primaria. Infancia y Aprendizaje, 33, 413-425.

Raven, J.C. (2005). Test de matrices progresivas. Escala coloreada, general y avanzada. Buenos Aires: Paidós.

Ricketts, J. (2011). Research review: Reading comprehension in developmental disorders of language and communication. Journal of Child Psychology and Psychiatry, 52, 1111-1123.

Stanovich, K. (1989). L'évolution des modèles de la lecture et d'apprentissage de la lecture. En L. Rieben y Ch. Perfetti (Eds.), Textes de base en psychologie. L'apprenti lecteur (pp. 43-59). Neuchatel-Paris: Delachaux et Niestlé.

Webster, P. y Plante, A. (1992). Effects of Phonological impairment on word, syllable, and phoneme segmentation and reading. Language, Speech and Hearing Services in Schools, 23, $176-182$.

Woodcock, R., Muñoz, A., Ruef, M. y Alvarado, C. (2005). Language survey-revised. Test Book- Spanish. Rolling Meadows: Riverside Publishing Company. 